\title{
Dissociation between scopolamine and mecamylamine during fear conditioning in rats*
}

\author{
AGU PERT and H. H. AVIS $\dagger$ \\ Experimental Medicine Branch, Biomedical Laboratory, Edgewood Arsenal, Maryland 21010
}

\begin{abstract}
State-dependent (dissociated) learning has been reported for both scopolamine (muscarinic anticholinergic) and mecamylamine (nicotinic anticholinergic). The purpose of this series of experiments was to ascertain whether dissociation would occur between these two anticholinergic compounds in a conditioned suppression task. In Experiment I, food-deprived rats were trained to drink sweetened condensed milk in a test chamber for 8 days. On Day 9 , all rats received a single electric shock in the same chamber $20 \mathrm{~min}$ after injections of scopolamine, mecamylamine, or saline. Three days later, subgroups were tested for conditioned suppression under the same drug condition, the other drug condition, or saline. Conditioned suppression was found in all groups except those trained under scopolamine or mecamylamine and tested under saline or the other drug condition. Experiment II controlled for some of the variables which may have produced dissociation in the first experiment. The findings are interpreted to imply the existence of two separate cholinergic (nicotinic and muscarinic) pathways which mediate fear conditioning.
\end{abstract}

Although the presence of both nicotinic and muscarinic cholingergic receptors in the CNS has received considerable substantiation recently from a number of diverse sources (Curtis \& Crawford, 1970; Domino, 1967, 1968; Schechter \& Rosecranz, 1971), few investigators have attempted to examine the behavioral significance of this differential coding of cholinergic CNS neurons. The possibility exists, for example, that muscarinic and nicotinic CNS circuits are selectively activated during specific behaviors.

One approach that can be employed to assess the significance of this differential nicotinic and muscarinic neurochemical coding of cholingergic CNS neurons is to analyze and compare the behavioral effects seen following the blockade of normal cholinergic activity by either antimuscarinic (e.g., scopolamine) or antinicotinic (e.g., mecamylamine) compounds. Both similarities and differences have appeared between the behavioral effects of these two classes of anticholinergic compounds in a variety of behavioral paradigms. Passive avoidance deficits, for example, have been reported in a number of different experimental settings following administration of either scopolamine or mecamylamine (Bohdanecky \& Jarvik, 1967; Calhoun \& Grant, 1972; Chiappetta \& Jarvik, 1969; Dilts \& Berry, 1967; Glick \& Greenstein, 1972; Goldberg, Sledge, Hefner, \& Robichaud, 1971). Acquisition of one-way active avoidance has been impaired by mecamylamine (Goldberg et al, 1971), an

\footnotetext{
*In conducting the research described in this paper, the investigators adhered to the "Guide for Laboratory Animal Facilities and Care" as promulgated by the Committee on the Guide for Laboratory Animal Resources, National Academy of Sciences-National Research Council. We are grateful to Margaret Griffin, William Lee, and George Maxey for their expert technical assistance. Requests for reprints should be sent to Agu Pert, Psychology Group, Experimental Medicine Branch,

Biomedical Laboratory, Edgewood Arsenal, Maryland 21010.

+Present address: Laboratory of Psychobiology, Langley Porter Neuropsychiatric Institute, San Francisco, California 94122
}

effect similar to that reported for scopolamine (Meyers, Roberts, Riciputi, \& Domino, 1964; Suits \& Isaacson, 1968). Differences, however, have appeared between the effects of these two drugs in the acquisition of two-way active avoidance (Bignami, Amarico, Frontale, \& Rosic, 1971; Oliverio, 1966, 1967; Suits \& Isaacson, 1968) and in habituation (Avis \& Pert, 1974).

This pattern of findings suggests that the cholingergic neural systems which mediate certain behaviors (e.g., two-way active avoidance or habituation) may have selective neurochemical coding (muscarinic or nicotinic). If this hypothesis is valid, then a disruption of cholinergic transmission by antimuscarinic compounds should, in fact, produce a profile of behavioral effects possibly quite different from that produced by antinicotinic compounds. Some behaviors, on the other hand, may not possess differentially coded nicotinic or muscarinic substrates. These behaviors would be affected presumably in a similar manner by either class of anticholingergic compounds.

Although similarities have appeared between the behavioral effects of scopolamine and mecamylamine, this does not necessarily imply that both compounds are affecting identical central systems. One way to ascertain whether similar or different systems are affected by both drugs is to test for dissociation between the two. Overton (1968b, 1971) has reviewed data which indicate that behaviors learned while an animal is drugged may appear conditional on that drug state. In effect, behaviors acquired under a drug state produced by a centrally acting drug are only manifested under that drug state and fail to transfer to the undrugged state or state produced by another class of compounds (state-dependent learning). If mecamylamine and scopolamine do produce different central states or affect different CNS systems, then it is conceivable that behaviors learned under one will not transfer to the 


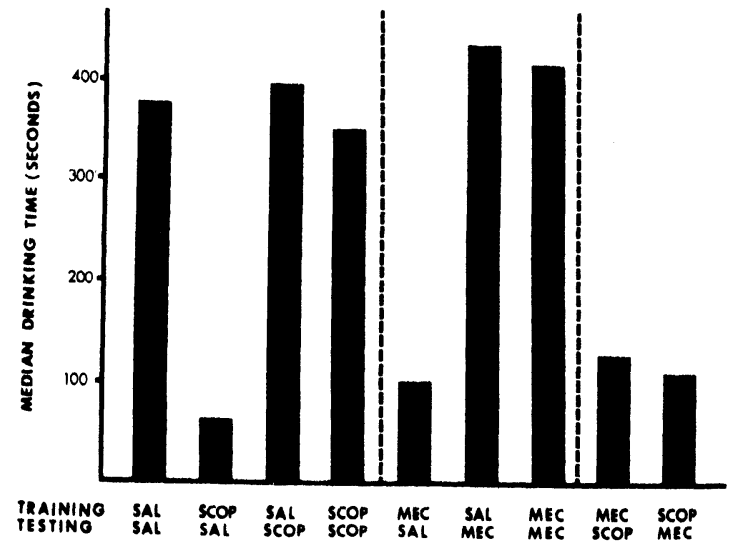

Fig. 1. Median drinking times for the various groups in the retention test of Experiment $I$. Training and retest conditions are indicated on the abscissa.

other, even though both compounds produce similar behavioral effects and state-dependent learning in a specific situation.

Glick and Greenstein (1972) have reported a study in which they found dissociation between mecamylamine and scopolamine in passive avoidance. Experiment I was designed to look for dissociation between these two compounds in fear conditioning, another paradigm which has been found to be sensitive to both (Avis \& Pert, 1974). ${ }^{1}$ The purpose of Experiment II was to provide some of the necessary controls for the cross-dissociation design of Experiment I.

\section{EXPERIMENT I}

\section{Method}

Subjects. Eighty-four male Sprague-Dawley rats were the Ss of this experiment. The animals were housed individually in the same room as the apparatus. A 23-h food-deprivation schedule was begun 5 days before the start of the experiment, and the animals were maintained on this schedule throughout the study. The lighting schedule of the experimental room was $14 \mathrm{~h}$ light and $10 \mathrm{~h}$ dark. The experiment was always conducted during the beginning of the light cycle.

Apparatus. The apparatus consisted of an aluminum chamber $(23 \times 23 \times 30 \mathrm{~cm})$ with a clear Plexiglas front and a stainless steel grid floor. A glass drinking tube (BRS No. DT-001), filled with a solution of 2 parts water and 1 part sweetened condensed milk (Borden's brand), was mounted behind one wall approximately $4 \mathrm{~cm}$ above the floor. In order to facilitate the measurement of discrete licks, the tip of the tube was recessed $.5 \mathrm{~cm}$ behind a small Plexiglas insert containing a $.8-\mathrm{cm}$ hole. Drinking was recorded with a BRS drinkometer amplifier (BRS No. DO-201). Appropriate equipment was located in a separate room to record the total time required by each animal to complete 100 licks. A timer started when the door of the sound-attenuating chamber containing the apparatus was closed and stopped when the animal had completed 100 licks.

Procedure. In the preliminary training phase, all rats were allowed to make 100 licks at the drinking tube each day for 8 days. An animal was removed from the apparatus if it failed to complete 100 licks in $10 \mathrm{~min}$. At the end of 8 days, rats that had mean drinking times above $200 \mathrm{sec}$ over the last 2 days were eliminated from the study. This criterion resulted in the attrition of three rats from the study.
On Day 9, all rats were put into the apparatus under the appropriate drug conditions described below. The drinking tube had been removed for this session. Fifteen seconds after being put into the apparatus, the rats received a 1-mA 1 -sec shock to the paws, using a Grason-Stadler scrambled shocker (No. E1064GS). The animals were immediately returned to their home cages following the shock. Three days later, they were tested for the retention of fear conditioning (conditioned suppression) by measuring the time required to complete 100 licks. Normal animals subjected to such training show a 200-400-sec increase in their drinking times when retested. The increased drinking times are due to freezing behavior which competes with drinking.

Prior to fear conditioning, the animals were divided into nine matched groups of nine rats each based on their mean drinking times over the last 2 preliminary training days. Four basic treatment categories were established: (1) A saline control group against which retention could be evaluated. The animals in this group were shocked under saline and tested under saline (SAL-SAL). (2) Scopolamine treatment subgroups, consisting of a group trained under scopolamine and tested under scopolamine (SCOP-SCOP), , another group trained under scopolamine and tested under saline (SCOP-SAL), and a final group trained under saline and tested under scopolamine (SAL-SCOP). (3) Mecamylamine subgroups that were treated exactly like the scopolamine groups (MEC-MEC, MEC-SAL, SAL-MEC). (4) Groups in which cross-dissociation could be evaluated (SCOP-MEC and MEC-SCOP).

All drugs were injected intraperitoneally at a volume of $1 \mathrm{cc} / \mathrm{kg} 20 \mathrm{~min}$ before the animals were put into the apparatus. Scopolamine hydrobromide was injected at a dosage of $1 \mathrm{mg} / \mathrm{kg}$ and mecamylamine $\mathrm{HCl}$ at a dosage of $5 \mathrm{mg} / \mathrm{kg}$. ${ }^{2}$ The vehicle for both drugs was normal saline.

\section{Results and Discussion}

Figure 1 summarizes the retention performance of all groups in terms of median drinking times. Inspection of this figure reveals an apparent impairment in conditioned suppression in the groups that were trained under either mecamylamine or scopolamine and tested under saline or the opposite drug condition (MEC-SAL, SCOP-SAL, MEC-SCOP, SCOP-MEC). Comparisons of the performance of each of these groups to the performance of the control group trained under saline and tested under saline (SAL-SAL) revealed significant differences with the Mann-Whitney $U$ test for all comparisons (SAL-SAL vs MEC-SAL, $\mathrm{U}=12, \mathrm{p}<.02$; SAL-SAL vs SCOP-SAL, $U=5, p<.02 ;$ SAL-SAL vs MEC-SCOP, $U=14, p<.02$; SAL-SAL vs SCOP-MEC, $U$ $=15, p<.05)$. All other groups failed to differ significantly from the SAL-SAL control (SAL-SAL vs SAL-SCOP, $U=33, p>.05$; SAL-SAL vs SCOP-SCOP, $\mathrm{U}=38, \mathrm{p}>.05 ;$ SAL-SAL vs SAL-MEC, $\mathrm{U}=29$, $\mathrm{p}>.05$; SAL-SAL vs SCOP-SCOP, $U=38, \mathrm{p}>.05$; SAL-SAL vs SAL-MEC, $U=29, \mathrm{p}>.05$; SAL-SAL vs MEC-MEC, $\mathrm{U}=34, \mathrm{p}>.05$ ). In addition, comparisons of the groups trained and tested under the same drug with their respective cross-dissociation group (i.e., SCOP-SCOP vs SCOP-MEC and MEC-MEC vs MEC-SCOP) were also found to be significant with the Mann-Whitney U test (SCOP-SCOP vs SCOP-MEC, U = $17, \mathrm{p}<.05$; MEC-MEC vs MEC-SCOP, $\mathrm{U}=13, \mathrm{p}<.02$ ).

Although antimuscarinic compounds have been reported to decrease water intake (Houser, 1970; Stein, 
1963), this factor could not have confounded the results of this experiment. First, the two cross-dissociation groups which were tested under either scopolamine (MEC-SCOP) or mecamylamine (SCOP-MEC) did not show an appreciable increase in drinking times. Second, we have previously shown (Avis \& Pert, 1974) that scopolamine and mecamylamine, at the dosages employed in this study, have no effect on the consumption of diluted sweetened condensed milk.

In part, some of the findings of this study replicate our earlier findings for scopolamine and mecamylamine in the identical paradigm (Avis \&.Pert, 1974). Again, both compounds were found to produce asymmetrical dissociation during fear conditioning and retention (i.e., lack of conditioned suppression in the groups trained under either mecamylamine or scopolamine and tested under saline and perfect retention of fear conditioning in the groups trained under saline and tested under either compound). The major findings of interest in this study, however, was the lack of transfer of fear conditioning between scopolamine and mecamylamine. This finding is particularly striking since the groups that were trained under scopolamine or mecamylamine and tested under the same drug condition demonstrated perfect conditioned suppression. The dissociation between scopolamine and mecamylamine strongly suggests that each of these compounds may be producing similar behavioral effects through different cholinergic systems of the CNS.

Although our findings indicate that mecamylamine and scopolamine may be affecting different central systems, two other possibilities, related to the stimulus properties of these compounds, exist which may also account for our results. These possibilities were tested in the next experiment.

\section{EXPERIMENT II}

State-dependent learning closely resembles stimulus generalization. A response or behavior learned in one drug state may fail to appear when that drug state is altered. This deficit is assumed to be due to the stimulus generalization decrement that occurs between the drug-produced stimuli which are present during training and their absence during subsequent testing. One extreme way in which to effect this stimulus change is to remove the drug state altogether during testing (e.g., Group SCOP-SAL of the first experiment). Another method would be to alter the degree of the drug state between training and testing by administering either a lower or a higher dose of the same drug during testing. Overton (1968a) has, in fact, demonstrated that a rat is capable of discriminating between two different doses of the same drug. This finding indirectly indicates the possibility of a stimulus generalization decrement occurring between two concentrations of the same drug.

The lack of transfer in the previous experiment between mecamylamine and scopolamine may have been caused by our failure to have properly equated the dosages between the two different anticholinergic compounds. The implicit assumption behind such reasoning is that total cholinergic blockade (whether nicotinic or muscarinic) is the critical variable that provides the necessary stimuli for state-dependent learning.

Another possibility is that the dissociation in the present experiment may have been due to the different peripheral effects produced by the two anticholinergic compounds. It is conceivable, for example, that the peripheral effects of either scopolamine- or mecamylamine-produced stimulus conditions differed enough from each other during training to prevent appropriate recall during testing due to a stimulus generalization decrement.

These two possibilities were evaluated in the following study.

\section{Method}

Subjects. Seventy male Sprague-Dawley rats were used as Ss in this experiment. They were housed and maintained in the same manner as those in the first experiment.

Apparatus. The apparatus was the same as that employed in the first experiment.

Procedure. The procedures in this study for training and testing the animals were identical to those employed in the preceding experiment, with the exception of some drug treatments described below. Three basic treatment categories were formed: (1) Three groups of animals provided the necessary controls for state-dependent learning under scopolamine. One group was trained under saline and tested under saline (SAL-SAL), the second group was trained under scopolamine and tested under saline (SCOP-SAL), and the third group was trained under scopolamine and tested under scopolamine (SCOP-SCOP). These groups provided the necessary controls against which to evaluate the presence of state-dependent learning in the other treatment groups. (2) Three groups of animals provided a test of the postulate that mecamylamine (at the dose employed in the first experiment) provided stimuli which were quantitatively different from those produced by scopolamine, thus producing dissociation. All three groups received scopolamine during training $(1 \mathrm{mg} / \mathrm{kg})$ and 1,5 , or $10 \mathrm{mg} / \mathrm{kg}$ of mecamylamine during testing (SCOP-MEC 1, SCOP-MEC 5, and SCOP-MEC 10, respectively). (3) The third treatment category consisted of only one group, which provided a test of the postulate that dissociation occurred in the first experiment between scopolamine and mecamylamine because of the differences in peripheral effects between these two anticholinergic compounds. This group was trained under scopolamine and tested under a combination of scopolamine $(1 \mathrm{mg} / \mathrm{kg})$ and hexamethonium $(5 \mathrm{mg} / \mathrm{kg})$, an antinicotinic drug that does not readily pass into the CNS. If the peripheral antinicotinic effects of mecamylamine are important in producing dissociation between scopolamine and mecamylamine, then it was to be expected that this group would show a decrement in conditioned suppression when compared to the group trained and tested under scopolamine (SCOP-SCOP).

The 70 animals were divided into seven equal groups at the end of drinkometer training. They were matehced on the basis of their mean drinking times over the last 2 training days.

\section{Results and Discussion}

The results of this study appear in Fig. 2 in terms of median drinking times during the retention testing. The performance of the SAL-SAL, SCOP-SAL, and 


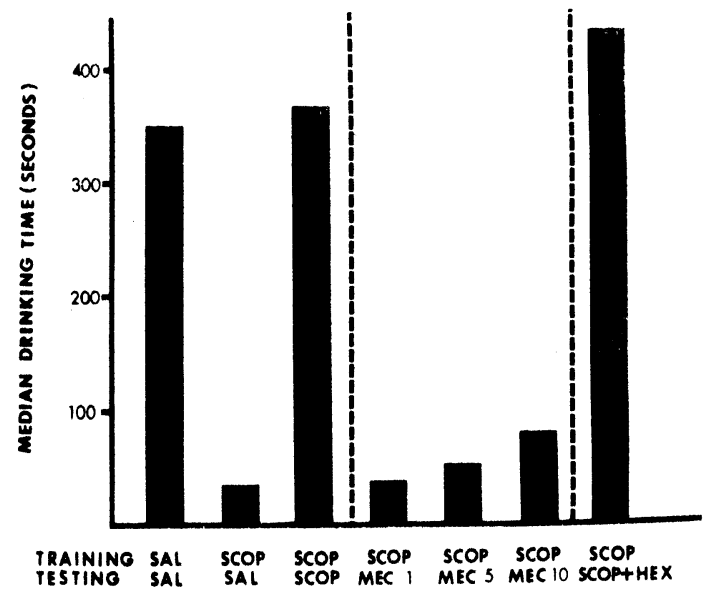

Fig. 2. Median drinking times for the various groups during the retention test of Experiment II. Training and retest conditions are indicated on the abscissa.

SCOP-SCOP groups of the present experiment was almost identical to that of the same groups in the previous experiment. The median drinking time of the SCOP-SAL group was again found to be significantly different from that of the SAL-SAL group, with the Mann-Whitney $U$ test $(U=10, p<.02)$ indicating poor retention of fear conditioning in the former group. The group trained under scopolamine and tested under scopolamine (SCOP-SCOP), on the other hand, again demonstrated perfect retention, since the drinking time for this group failed to differ significantly from thatof the SAL-SAL group $(U=44, p>.05)$. The three groups trained under scopolamine and tested under 1,5 , and $10 \mathrm{mg} / \mathrm{kg}$ of mecamylamine (SCOP-MEC 1, SCOP-MEC 5, or SCOP-MEC 10) all failed to recall the fear conditioning, since none of the groups was found to be significantly different from the SCOP-SAL group (SCOP-SAL vs SCOP-MEC 1, U $=44, \mathrm{p}>.05$; SCOP-SAL vs SCOP-MEC 5, U = 31, p > .05; SCOP-SAL vs SCOP-MEC 10, $U=28, p>.05$ ) but did differ from the SAL-SAL group (SAL-SAL vs SCOP-MEC $1, \mathrm{U}=10$, $\mathrm{p}<.002$; SAL-SAL vs SCOP-MEC $5, \mathrm{U}=23, \mathrm{p}<.05$ ). These findings lend strong support to the contention that a possible difference in degree of total cholinergic blockade by mecamylamine as compared to scopolamine in the first experiment was not the critical variable in producing dissociation between the two anticholingergic compounds.

The group trained under scopolamine and tested under a combination of scopolamine and hexamethonium (SCOP-SCOP+HEX) demonstrated perfect conditioned suppression despite the different peripheral drug effects present during testing. The performance of this group failed to differ significantly from that of the SCOP-SCOP group $(U=35, p>.05)$. Before any conclusions can be formulated from this finding, however, it must be shown that the seemingly perfect conditioned suppression exhibited by the
SCOP-SCOP+HEX group is not the result of a general impairment in performance produced by the scopolamine-hexamethonium drug combination. This was done by training two groups of 10 animals each to drink sweetened condensed milk for 8 days in the same apparatus and then testing each of thesygroups under either saline or scopolamine $(1 \mathrm{mg} / \mathrm{kg})$ plus hexamethonium $(5 \mathrm{mg} / \mathrm{kg})$. The saline group had a median drinking time of $42.6 \mathrm{sec}$, while the group tested for drinking under scopolamine-hexamethonium had a median drinking time of $56.8 \mathrm{sec}$. The increase in drinking time produced by the drug mixture was not found to be significant, employing the Mann-Whitney U test $(p>.05)$. Apparently, the conditioned suppressing found in the SCOP-SCOP+HEX group was determined by the retention of fear and not by a general performance decrement.

These findings indicate that the peripheral antinicotinic effects produced by mecamylamine in the first experiment were not critical in producing dissociation between scopolamine and mecamylamine. Peripheral drug effects produced by drugs that do not readily enter the brain have in fact been found to be relatively ineffective in establishing discriminative control over behavior (Overton, 1971). It appears unlikely that the peripheral effects of drugs play any significant role in state-dependent phenomena.

On the basis of the above findings, it is tentatively concluded that neither possible quantitative differences between scopolamine and mecamylamine nor the peripheral effects of these two compounds played a significant role in producing dissociation. It is tempting to suggest that the dissociation was due to the effects of the two anticholinergic compounds on differentially coded (i.e., nicotinic and muscarinic) neuronal circuits that mediate fear conditioning.

\section{GENERAL DISCUSSION}

Although mechanisms based on stimulus generalization decrement have been proposed (Overton, $1967,1971)$ to account for findings from studies investigating state-dependent learning phenomena, it is clear that this type of mechanism is insufficient by itself to account for the asymmetrical dissociation found in this experiment with scopolamine and mecamylamine (i.e., the findings that animals trained under either scopolamine or mecamylamine and tested under saline exhibited retention deficits, while animals that were trained under saline and tested under either scopolamine or mecamylamine did not).

Berger and Stein (1969) have recently proposed a hypothetical model to account for the asymmetrical dissociation produced by scopolamine in fear conditioning. We have adopted and modified this model to account for our findings in Experiment I. Figure 3 depicts the hypothetical neuronal events and conditions 
Fig. 3. Hypothetical neuronal model depicting the events underlying asymmetrical dissociation found with scopolamine and mecamylamine and the dissociation between the two compounds in fear conditioning.

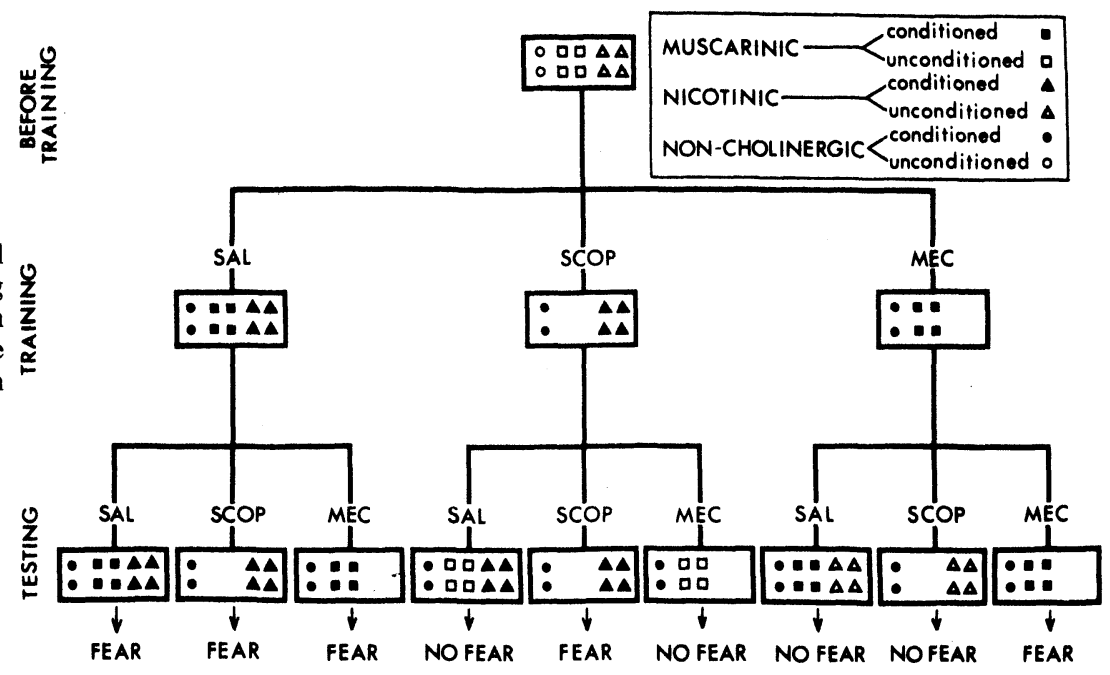

before, during, and after fear conditioning in this experiment.

However, before this model is described in any greater detail, it will be necessary to first analyze the entire experimental situation from the start to determine how the various components contribute to the appropriate recall or to the retention deficit during retention testing.

Two stages of conditioning appear to exist in this experimental paradigm. In Stage 1, subsets of nicotinic, muscarinic, and noncholinergic elements are conditioned to the drinking of sweetened condensed milk from the tube. Since the duration of training is long in this stage, conditioning of these elements is probably strong. During Stage 2 (fear conditioning), the functional elements may be assumed to become conditioned to shock. This conditioning is probably weak, since the session is brief. During retention testing, if a set of elements is functional which has not been fear conditioned, it could be assumed to be behaviorally dominant over any fear-conditioned elements which are only weakly conditioned.

With these considerations in mind, it is now possible to analyze the findings from the first experiment.

In Fig. 3, the large rectangles enclosing the small circles, squares, and triangles are assumed to represent the primary neuronal system that is involved in fear conditioning. This system, it should be noted, has previously undergone conditioning to drinking in the chamber. The small circles, squares, and triangles within the rectangles depict the individual neuronal elements (e.g., synapses or receptors). These elements are further postulated to fall into three basic categories: noncholinergic, nicotinic, and muscarinic. ${ }^{3}$ The nicotinic elements are presumably blocked by only antinicotinic agents (e.g., mecamylamine), whereas the muscarinic elements are blocked only by antimuscarinic agents (e.g., scopolamine). The noncholingergic elements, on the other hand, are resistant to the effects of either scopolamine or mecamylamine. In order to properly evaluate the effects of the various drug treatments on fear conditioning in the context of this model, it will be necessary to analyze each of the three treatment categories pictured in Fig. 3 separately: (1) In all groups trained under saline, the neuronal elements that mediate fear are conditioned during the training session (conditioning depicted by filling in the small circles, squares, and triangles). When these groups are tested for conditioned suppression under saline, scopolamine, or mecamylamine, they exhibit perfect retention, since all or some of the elements that were originally fear conditioned are still functional and there are no elements present which were originally conditioned to drinking and not fear conditioned. (2) In the groups trained under scopolamine, all the muscarinic elements were functionally blocked during training and therefore failed to undergo conditioning at all (depicted in Fig. 3 by the absence of muscarinic elements during fear training). During testing for conditioned suppression, the animals that were trained under scopolamine and tested under saline show an apparent deficit in retention presumably because the fear-unconditioned muscarinic elements which are present and functional during the retest had undergone strong conditioning to drinking in the first stage and are now behaviorally dominant and able to override the emotional behavior produced by the fear-conditioned nicotinic and noncholinergic elements. Blocking the nicotinic elements of the animals trained under scopolamine can only exaggerate this retention deficit, since now, in addition to the presence of dominant competing behavior during testing, there is also an absence of some of the elements (nicotinic) that were conditioned during fear training. Blocking the muscarinic elements during testing, however, can produce perfect retention, since only those elements that were conditioned during fear training are functional and activated during testing. (3) A similar analysis applies to the groups trained under mecamylamine and tested under saline, scopolamine, or mecamylamine. Again, the dissociation between the two anticholingergic drugs is predicted on the basis of the presence of 
fear-unconditioned nicotinic elements during testing.

A close examintion of this model reveals that the groups which exhibited a deficit in conditioned suppression have one similarity: they all have dominant fear-unconditioned elements present and functional during the retest which contribute to behavior (drinking) that competes with freezing or fear. The presence of dominant unconditioned pathways in the CNS during retention testing may be also one of the underlying mechanisms behind the deficits that are seen following training under a variety of drugs in a number of different experimental paradigms. Whether this model is indeed applicable to other situations and drugs remains to be disclosed by further experimentation.

The findings from this investigation provide further evidence for the existence of nicotinic and muscarinic cholinergic circuitry in the CNS and, in addition demonstrate that both types of cholingergic pathways underlie fear conditioning. Additional studies, employing other behavioral paradigms, are needed to further elucidate the functional roles of nicotinic and muscarinic coding in the CNS.

\section{REFERENCES}

Avis, H. H., \& Pert, A. A comparison of the effects of muscarinic and nicotinic anticholingergic drugs on habituation and fear conditioning in rats. Psychopharmacologia, 1974, 34, 209-222.

Berger, B. D., \& Stein, L. Asymmetrical dissociation of learning between scopolamine and WY 4036, a new benzodiazepine tranquilizer. Psych oph armacologia, 1969, 14, 351-358.

Bignami, G., Amarico, L., Frontale, M., \& Rosic, N. Central cholingergic blockade and two-way avoidance acquisition: The role of response disinhibition. Physiology \& Behavior, 1971 7, 461-470.

Bohdanecky, Z, \& Jarvik, M, E. Impairm ent of one-trial passive avoidance learning in mice by scopolamine, scopolamine methylbromide, and physostigmine. International Journal of Neuropharmacology, 1967, 6, 217-222.

Calhoun, W. H., \& Grant, M. J. Preinjection time of scopolamine and step-down latency in mice. Psychonomic Science, 1972. 26, 39-40.

Carlton, D. L., \& Vogel, J. R. Studies of the amnesic properties of scopolamine. Psychonomic Science, 1965, 3, 261-262.

Chiappetta, L., \& Jarvik, M. E. Comparison of learning impairment and activity depression produced by two doses of cholingergic blocking agents. Archives Internationales de Pharmacodynamie et de Therapie, 1969, 179, 161-166.

Curtis, D. R., \& Crawford, J. M. Central sy naptic transmission-microelectrophoretic studies. Annual Review of Pharmacology, 1969, 9, 209-240.

Dilts, S. L., \& Berry, C. A. Effects of cholinergic drugs on passive avoidance in the mouse. Journal of Pharmacology \& Experimental Therapeutics, 1967, 158, 279-285.

Domino, E. G. Electroencephalographic and behavioral arousal effect of small doses of nicotine: A neuropsychopharmacological study. Annals of the New York Academy of Sciences, 1967, 142, 216.
Domino, E. F. Role of the central cholinergic system in wakefulness, fast wave sleep and no-go behavior. In: The present status of psychotropic drugs. Proceedings of the VI International Congress of the C.I.N.P. Excerpta Medica International Congress, 1968.

Glick, S. D., \& Greenstein, S. Differential effects of scopolamine and mecamylamine on passive avoidance behavior. Life Sciences, 1972, 11, 169-179.

Goldberg, M. E., Sledge, K., Hefner, M., \& Rabichaud, R. C. Learning impairment after three classes of agents which modify cholinergic function. Archives Internationales de Pharmacodynamie et de Therapie, 1971, 193, 226-235.

Houser, V. P. The effects of adrenergic and cholinergic agents upon eating and drinking in deprived rats. Psychonomic Science, 1970, 20, 153-155.

Meyers, B., Roberts, K. H., Riciputi, R. H., \& Domino, E. F. Some effects of muscarinic blocking drugs on behavior and electrocardiogram. Psychopharmacologia, 1964, 5, 289-300.

Oliverio, A. Effects of mecamylamine on avoidance conditioning and maze learning of mice. Journal of Pharmacology \& Experimental Therapeutics, 1966, 154, 350-356.

Oliverio, A. Contrasting effects of scopolamine on mice trained simultaneously with two different schedules of avoidance conditioning. Psychopharm acologia, 1967, 11. 39-51.

Overton, D. A. Visual cures and shock sensitivity in the control of T-maze choice by drug conditions. Journal of Comparative \& Physiological Psychology, 1968a, 66, 216.

Overton, D. A. Dissociated learning in drug states (state-dependent learning). In D. H. Efron et al (Eds.), Psychopharmacology. A review of progress. PHS Publication No. 1836. Washington, D.C: U.S. Government Printing Office, $1968 \mathrm{~b}$.

Overton, D. A. Discriminative control of behavior by drug states. In T. Thompson and R. Pickens (Eds.), Stimulus properties of drugs. New York: Appleton-Century-Crofts, 1971. Pp. 87-110.

Schechter, M. D., \& Rosecranz, J, A. Behavioral evidence for two types of cholinergic receptors in the CNS. European Journal of Pharmacology, 1971, 15, 375-378.

Stein, L. Anticholinergic drugs and the central control of thirst. Science, $1963,139,46-48$.

Suits, E, \& Isaacson, $R$, L. The effects of scopolamine hydrobromide on one-way and two-way avoidance learning in rats. International Journal of Neuropharmacology, 1968, 7, 441-446.

\section{NOTES}

1. Goldberg, Sledge, Hefner, and Robichaud (1972) have reported a study in which they were able to differentiate the effects of scopolamine and mecamylamine in a fear-conditioning paradigm. This experiment, however, contained a number of methodological flaws which made interpretation of the results difficult. See Avis and Pert (1974) for a full critical treatment of this study.

2. The dosages of mecamylamine and scopolamine were chosen on the basis of previous behavioral experiments with these compounds (Avis \& Pert, 1974; Carlton \& Vogel, 1965; Dilts \& Berry, 1967).

3. At this point, our model departs from the one proposed by Berger and Stein by making the assumption that the cholinergic system which mediates fear conditioning can be subdivided into muscarinic and nicotinic components. This assumption is based on the findings that both nicotinic and muscarinic blockade are equally effective in disrupting fear conditioning.

(Received for publication March 28, 1973; revision received January $10,1974$. 